\title{
THE EFFECT OF A CORONAL EXPANSION ON THE COMPUTATION OF AN ELECTRON TEMPERATURE FROM EMISSION-LINE INTENSITIES*
}

\author{
WERNER M. NEUPERT
}

\begin{abstract}
Certain assumptions made in the calculation of a coronal electron temperature from the relative populations in two stages of ionization of an element are examined, with the conclusion that this calculation may not be valid for the particular case of the solar corona, yielding a fictitiously low value if there is an outward expansion of ions from the base of the corona into a region of higher electron temperature. A non-equilibriım formulation for the distribution of ions is presented that may better describe the actual ion distribution in the corona. An approximate calculation shows that an outward radial velocity which is small compared with the average thermal velocity of the ions is sufficient to explain the apparent observed increase in height of the maxima of emission of the Fe ion sequence with increasing stage of ionization.
\end{abstract}

\section{INTRODURTIÚN}

Past observations of the solar corona have resulted, with considerable consistency, in a discrepancy between the electron temperature of the "quiet" or "normal" corona, undisturbed by centers of activity, as obtained (a) from the electron density gradient inferred from scattering of photospheric light by electrons and (b) from ionization theory. The first method yields values of about $1.6 \times 10^{6}{ }^{\circ} \mathrm{K}$, whereas the second, from the ratio $\mathrm{Fe} \mathrm{xIv} / \mathrm{Fe} \mathrm{x}$, gives values half as large. Recent measurenents by Orbiting Solar Observatory-I indicate that the latter method may lead to inconsistent results, for the ratio $\mathrm{Fe} \mathrm{xvi} / \mathrm{Fe} \mathrm{xv}$, as $\mathrm{mr}$ ?sured by the satellite, implies an average electron temperature of $1.7-1.8 \times 10^{\circ}{ }^{\circ} \mathrm{K}$ (Neupert 1963). In addition to these values for the electron temperature, a high value of the kinetic temperature, of the order of $2.0 \times 10^{6}{ }^{\circ} \mathrm{K}$, is obtained from observed line-broadening if it is assumed that the broadening is due entirely to thermal motions. It has often been suggested that the electron temperature computed from the ratio $\mathrm{Fe}$ $\mathrm{xIv} / \mathrm{Fe} \mathrm{x}$ is the more accurate value, while that

*Published in The Astrophysical Journal, 139(3):435-040, April $1,1064$. (kinetic) temperature determined from line-broudening is inaccurate because of the presence of nonthermal motions. Although this possibility exists, it has yet to receive obseivational verification.

Line-broadening measurements also suggest that any temperature gradient in the quiet corona is quite small at heights between $r=1.03 R \odot$ ( $R \odot$ being the solar radius) and a few solar radii, being negative and of the order of $3^{\circ} \mathrm{K}$ per $\mathrm{km}$ for the Fe XIV (5303 $\AA$ ) line (Billings and Lilliequist 1963). Under such circumstances it becomes difficult to account for the apparent simultaneous existence of low stages of ionization, such as $\mathrm{Fe} \mathrm{x}$ and $\mathrm{Fe} \mathrm{xI}$, and also a stage as high as $\mathrm{Fe} \mathrm{XvI}$, observed by CSO-I to be the emitter of two of the more intense emission lines of the extreme ultraviolet spectrum between $170 \AA$ and $400 \AA$, even in the absence of visible centers of activity. The suggestion has been made (Shklovsky 195i) that limb oi iservations may include regions of differing electron temperature, thereby giving the spectrum a misleading appearance. Such an argument is without doubt correct near centers of activity which manifest an increase in electron temperature by the appearance of the $\mathrm{Ca} \mathrm{XV}$ $(5694 \AA)$ line. However, for the undisturbed corona, the existence of regions having the 
required range of electron temperatures would require tangential temperature gradients considerably greater than the radial gradient reported by Billings and Lilliequist.

Since it is the ionization theory which leads to an electron temperature considerably less than that obtained by other observational methods, it is of interest to re-examine certain assumptions made in the theory in light of current knowledge of the physical state of the quiet corona. It is the purpose of this paper to suggest that a combination of physical circumstances, at least in the quiet solar corona, may a!ter the distribution of ions in the various stages of ionuzation, thereby making the ionization theory invalid as a means of computing an electron temperature for the coronal plasma, and also permitting a broader distribution of ions than is allowed by ionization theory in a nearly isothermal corona. A steady state non-equilibrium formulation, valid for an optically thin plasma, is proposed which appears to be more compatible not only with present optical information about the corona at visible wavelengths but also with certain observations in the extreme ultraviolet spectrum made from rockets and satellites.

\section{EFFECT OF A CORONAL EXPANSION}

A combination of two physical conditions may lead to a discrepancy in the coronal temperatures derived from line intensities and from line-broadening. These two factors are (1) an average time between ionizations (of heavy ions) which is large compared with the time for equipartition of energy between the ions and the coronal plasma; (2) a mass motion of ions through a region having a high electron-temperature gradient in a period of time equal to or less than the above-mentioned time between ionizations. We will first consider the existence of each of these conditions in the lower corona and then discuss their influence on a theory which assumes ionization equilibrium.

An estimate of the time between ionizations may be obtained from ionization rates iron for ions computed from cross-sections given by Elwert (Massey 1956). These rates increase with increasing electron temperature, from $7.4 \times 10^{-13}$ $\mathrm{sec}^{-1} \mathrm{~cm}^{3}$ at $0.8 \times 10^{8}{ }^{\circ} \mathrm{K}$ to $1.95 \times{ }_{1} 0^{-11} \mathrm{sec}^{-1} \mathrm{~cm}^{3}$ at $2.0 \times 10^{8}{ }^{\circ} \mathrm{K}$ for $\mathrm{Fe} \mathrm{xIII}$, and decrease with increasing stage of ionization from $2.5 \times 10^{-11} \mathrm{sec}^{-1}$ $\mathrm{cm}^{3}$ for Fe $\mathrm{xI}$ to $3.5 \times 10^{-12} \mathrm{sec}^{-1} \mathrm{~cm}^{3}$ for $\mathrm{Fe} \mathrm{XV}$ at $1.6 \times 10^{6}{ }^{\circ} \mathrm{K}$. These rates are higher (by a factor of 4 or more) than those used by Firor and Zirin (1962) but slightly lower than those used by Seaton (1962). We will assume, as do Firor and Zirin, that the dependence of ionization rates on the number of valence electrons is small and may be neglected. A measure of the time between successive ionizations (disregarding recombinations) is then given by

$$
\tau_{\text {ion }}=\frac{1}{N_{e} S(T)}
$$

where $N_{0}$ is the electron density and $S(T)$ is the ionization rate in $\mathrm{sec}^{-1} \mathrm{~cm}^{3}$. Taking a value for $i_{\text {. }}$ of $2.0 \times 10^{8} \mathrm{~cm}^{-3}$ and a value for $S(T)$ of $1.0 \times 10^{-11} \mathrm{sec}^{-1} \mathrm{~cm}^{3}$, one finds that a time interval of the order of 500-1000 sec (one to two time constants) is required to ionize, without recombinations, in assemblage of ions from stage $i$ (taken as Fe xIII) to toge $i+1$ in the lower corona at an electron t....,erature of $1.6 \times 10^{6}{ }^{\circ} \mathrm{K}$, a value consistent with that obtained from the electrondensity gradient. On the other hand, the time required to establish equipartition of energy between protons and heavy ions in the coronal plasma is considerably shorter. This characteristic time can be computed from equations given by Spitzer (1956) and is of the order of $2.0 \mathrm{sec}$ for Fe xIII-proton equilibrium at an electron (and, approximately, proton) density of $2.0 \times 10^{8} \mathrm{~cm}^{-3}$ and a temperature of $1.6 \times 10^{\circ} \mathrm{K}$. It is of interest to note that the formula predicts the heavy ionproton equipartition time to be less than that for proton-proton equipartition by a factor of 2 (Fe XI) to 4 (Fe xvI) because of the high charge of the heavy ions. The time for equipartition is again inversely proportional to the electron $c$ snsity, leading to the relation

$$
\tau_{\text {lon }} / \tau_{\text {equi }} \sim \text { constant } \gg 1
$$

for a given ion anywhere in the solar corona.

In considering the second of the two conditions stated above we must restrici ourselves to regions of low magnetic field, thus permitting an expansion of the corona which results in the corpuscular stream deduced by Biermann (1951) from his wc on comet tails and was more recently discussed by Parker (1960). Using the continuity equntion one can compute the average solar-wind 
velocity at any height in the atmosphere once its velocity and particle density at another height have been defined. Using as initial parameters (Parker 1961) a proton density of $1.8 \times 10^{7} \mathrm{~cm}^{-3}$ and a solar-wind velocity of $66 \mathrm{~km} / \mathrm{sec}$ at $r$ $=1.43 R \odot$ one then finds, near the base of the corona, assuming an electron density of $2.0 \times 10^{8}$ $\mathrm{cm}^{-3}$, a velocity of about $12 \mathrm{~km} / \mathrm{sec}$. A recent calculation by Billings and Lilliequist produces a value about half as great. One direct measurement of the solar wind in the lower corona, a radar observation, has been made which yields a velocity similar to the computed values. The Massachusetts Institute of Technology El Campo station has observed, at $38 \mathrm{Mc}$, a Doppler shift of $4 \mathrm{kc}$, corresponding to a radially outward velocity of the reflecting electrons of $16 \mathrm{~km} / \mathrm{sec}$ (James 1963). Knowing the electron density at which a critical frequency is reached, one may compute the velocity at other densities using the continuity equation. At an electron density of $2.0 \times 10^{8} \mathrm{~cm}^{-3}$ one then obtains $3.5 \mathrm{~km} / \mathrm{sec}$. It 1 . , t be pointed out that these velocities are at variance with those obtained by extrapolation of recent observations made by Mariner II between the orbits of Venus and Earth. From this satellite data one would predict a wind, for the quiet sun, of only 0.06 $\mathrm{km} / \mathrm{sec}$ in the lower corona. It remains to be seen, however, whether an extrapolation over nearly 1 a.u. using a limited sampling of the interplanetary plasma is vaiid. For this reason the radar results will be used in this paper. Using a solar-wind velocity of several kilometers per second, one concludes that an ion may travel radially outward a distance of $10^{3}-10^{4} \mathrm{~km}$ in the lower corcna before undergoing further ionization.

The result of fulfilling both of the above conditions is that an ion, in passing through a region having a steep positive electron-temperature gradient, very quickly attains a thermal velocity characteristic of the ', ler temperature (condition 1) but carries 'vith it, ins a much longer time and radial distance, a hisiory of any previous ionization equilibrium (condition 2). Under such circumstances no theory which postulates equilibrium between ionization and recombinations can furnigh a valid value for the electron temperature of the region even if the predominant ionization mechanism is electron impact, and recombination is by radiative capture. A kinetic tempersture derived from line-broadening may still be valia, however. Because of the large increase in temperature over a distance of $10000 \mathrm{~km}$ or less in the lower solar corona, it appears that the situation here described may well occur throughout most of the region of thi corona from which radiation is observed.

At any height in the corona one must then replace the equilibrium equation (Woolley and Allen 1948),

$$
N_{i} N_{e} S_{i}^{i+1}(\tilde{i})=N_{i+1} N_{\odot} Q_{i+1}^{i}(T),
$$

where $Q(T)$ is the recombination rate in $\sec ^{-1}$ $\mathrm{cm}^{3}$, with the more general equation

$$
\nabla \cdot F_{i} \gtreqless 0,
$$

where $F_{i}$ is the flux of ions, in stage $i$, of each element. In general,

$$
\begin{aligned}
& \left.\nabla \cdot F_{i}=\text { (ionizations from } i-1\right) \\
& \quad-(\text { recombinations to } i-1) \\
& +(\text { recombinations from } i+1)
\end{aligned}
$$$$
\text { - (ionizations from } i \text { ). }
$$

For an optically thin corona of low density in which photoionizations and three-body recombinations are assumed to be negligible one obtains

$$
\begin{gathered}
\nabla \cdot F_{i}=N_{i-1} N_{e} S_{i-1}^{i}(T)-N_{i} N_{e}\left[Q_{i}^{i-1}(T)+S_{i}^{i+1}(T)\right] \\
+N_{i+1} N_{d} Q_{i+1}^{i}(T),
\end{gathered}
$$

for each stage of ionization, $i$, of each element present, together with

$$
N(r)=\sum_{i} N_{i}(r),
$$

where $N_{1}$ is the number density in each stage of ionization. The summation is made over all stages of ionization of the particular element being considered.

For a spherically symmetric corona expanding with velocity of magnitude $v(r)$ we have, for the total flux of a particular ion crossing a shell of radius $r$,

$$
F_{2}=4 \pi r^{2} N_{1}(r) v(r)
$$

By requiring a steady-state outward flow, all ions moving outward with the same average velocity, we obtain

$$
F_{0}=\Sigma F_{1} \approx 4 \pi r^{2} N_{6}(r) v(r)=\text { constant } .
$$


In this case the summation includes all elements present. Using the notation $P_{i}$, where $P_{i}$ is the relative population in each stage of ionization $i$,

we obtain

$$
P_{i} \equiv \frac{N_{i}(r)}{N r)}
$$

$$
\begin{aligned}
\frac{d}{d r} P_{t}=\frac{N_{\ell}^{2}}{F_{0} / 4 \pi r^{2}}\left\{P_{t-1} S_{t-1}^{t}(T)-P_{i}\right. & {\left[Q_{i}^{i-1}(T)\right.} \\
& \left.\left.+S_{i}^{t+1}(T)\right]+P_{t+1} Q_{t+1}^{i}(T)\right\}
\end{aligned}
$$

for each element and each stage of ionization.

We note that this equation is valid for any local areas, e.g., plages, in which the outward expansion may be different from that of the undisturbed corona, since the coefficient of the right-hand side can be evaluated locally in the corona. This equation is similar in form to that obtained by Burton and Wilson (1961) for a fully contained plasma except for a change in the independent variable from time to position and a change in the coefficient of the right-hand side An analytic solution is possible under the assumption that only three adjacent stages of ionization are significantly changing for any value of the independent variable, all lower stages having reached a steady-state reln' ve population and all higher states having yet to be populated. Although such an assumption is apparently valid for the plasma treated by Burton and Wilson and permits an analytic solution to be obtained for any stage of ionization, this is not the case for the solar atmosphere. In the latter, ions in the lowest stages of ionization may exist in regions of high optical thickness where the equation given is not even approximately valid. In order to apply the equation to the corona, where it is valid, one therefors requires boundary conditions at the base of the corona where more than three stages of ionization may already be significantly populated. At this boundary the solar atmosphere exhibits considerable fine structure, on which insufficient data are available for a precise evaluation of the equations.

However, it is possible to discuss the general behavior of the system by considering the expansion of an assemblage of ions from a region of low electron temperature of $5 \times 10^{8}{ }^{\circ} \mathrm{K}$ to a hotter region of $1.6 \times 10^{\circ}{ }^{\circ} \mathrm{K}$, the change in electron temperature taking place over a distance of only a few thousand kilometers. Examination of the equation for the predominant stage of ionization present at $5 \times 10^{5}{ }^{\circ} \mathrm{K}$ indicates that its relative population will decrease, in the absence of recombinations, by a factor of $1 / e$ in a distance of approximately

$$
\frac{F_{0}}{4 \pi r^{2}} \frac{1}{N_{e}^{2} S(T)}
$$

which is of the order of $10^{3} \mathrm{~km}$. Higher stages of ionization will become more populated with increasing height above the photosphere, reaching an ionization equilibrium characteristic of the higher temperature only in a distance of a few tens of thousands of kilometers. The reliability of this conclusion depends, of course, on the validity of the data we have used. If the solar proton measurements of Mariner II are used, rather than the radar measurement, together with the higher ionization rates of Seaton, the effect being discussed here will be entirely regligible.

\section{SOMPARISON WITH OBSERVATIONS}

A small amourt of observational evidence is available which appears to be consistent with the foregoing arguments. Beginning with the lower stages of ionization observed in the corona, it has been found, for the undisturbed corona, that $(a)$ the maximum surface brightness of Fe XI emission occurs below $10000 \mathrm{~km}$ above the photosphere (February 25, 1952 eclipse) (Athay and Roberts 1955), (b) the rnaximum surface brightness of $\mathrm{Fe}$ XIv occurs (statistically) at about $18000 \mathrm{~km}$ (Petri 1952), (c) the intensity of the Fe xv line at $7059 \AA$ decreases much more slowly with distance from the limb than do the lines of $\mathrm{Fe} \times(6374 \AA)$ and Fe xI (7891 $\AA$ ) (February 15, 1961, eclipse) (Wlerick and Fehrenbach 1961). This observation supports those of Allen (1946) who noted that the equivalent widths of coronal lines with relatively low ionization potentials showed a faster decrease with height above the photosphere than did lines of high ionization potential. $\mathrm{Ba}$ ied on the observation by OSO-I that the intensity of emission from $\mathrm{Fe}$ xvI requires emission from 0.1 to 0.5 of the solar corona, one might hypothesize, in accurdance with the model being presented here, that the maximum in surface brightness of $\mathrm{Fe}$ XVI occurs at about $40000 \mathrm{~km}$. 
Because of the rapidly decreasing electron density with height in the lower corona, the maximum in intensity of an ion may be very broad and need not be coincident with the inaximum in relative population of the ion.

The above observations are, of course, partially consistent with a coronal model, based on ionization equilibrium, which incorporates a positive temperature gradient rutward from the chromssphere. Such a model could not be employed beyond $1.03 R \odot$, however, since at distances greater than this the corona exhibits a negative temperature gradient, in contradiction to what would be expected from observation (c).

It is of interest to apply the arguments which have been developed to these observational data, thereby estimating the radial ion velocity in the lower corona. Assuming an average time between ionizations of $500 \mathrm{sec}$ for stages $\mathrm{Fe} \mathrm{XI}-\mathrm{xIV}$, we obtain, for the average radial velocity of these ions

$$
\bar{v}=\frac{10000}{1500}=6.6 \mathrm{~km} / \mathrm{sec} .
$$

Taking recombinations into account, we can reduce this velocity but by an amount estimated to be no more than 10 to 30 percent. This velocity is comparable to the solar-wind velocity in the lower corona inferred from radar observations. By cumparison, the average thermal velocity for Fe ions at $1.6 \times 10^{8}{ }^{\circ} \mathrm{K}$ is $15 \mathrm{~km} / \mathrm{sec}$. Since only limb observations of the corona are possible at visible wavelengths, little shift in the forbidden emission lines due to the outward ion velocity should be observable.

\section{CONCLUSION}

It appears that even at the low electron densities found in the corona a computation of the electron temperature based on ionization theory may be invalid in regions of outward expansion through an electron-temperature gradient. Such a condition may be responsible, in part, for the difference in coronal temperatures obtained from ionization theory and from line-broadening measurements. Its eventual importance will depend on bet ter estimation of ionization and recombination rates and the velocity of the solar wind. The presence of a coronal expansion does not rule out the existence of turbulence in the quiet corona, which may still account for the difference in temperatures obtained from electron-density gradients and from Doppler broadening of coronal lines.

\section{REFERENCES}

Allen, C. W. 1946, N.M., $106,137$.

1961, Proceedings of IAU Symposium No. 16, ed. J. W. Evans (New York:Academic Press), p. 1.

Athay, R. G., and Roberts, W. O. 1955, Ap.J., 121, 231. Biermans, L. 1951, Zs. f. A p., 29, 274.

Br.lings, D. E., and Lilliequist, C. G. 1963, $A$ p. J., $137,16$.

Burtoiv, W. M., and Wilson, R. 1961, Proc. Phys. Soc., $78,1416$.

Firor, J., and Zirin, H. 1962, Ap. J., 135, 122.

JAMEs, J. 1963, private communication.

Massey, H. S. W. 1956, Hdb. d. Phys., Vol. 36, ed. S. Flügge (Berlin:Springer-Verlag), p. 307.

Neupert, W. M. 1063, Space Research IV (to be published).

Parker, E. N, 1969, .1p. J., 132, 821.

1961, Space Astrophysics, ed. W. Liller (New York: McGraw-Hill Book Co.), p. 157.

Petri, W. 1952, Forschungsber. Ob . Wendelstein, Vol. 2.

SeAton, M. J. 1962, Observatory, 82, 111.

Shкцorsky, I. S. 1951, Izv. Krymsk. Ap. Obs,, 6, 105.

SpITzer, L. 1956, Physics of pully lonized Gases (New York:Interscience Publishers).

Weerick, G., and Fehrenbach, C. 1961, Proceedinge of IAU Symposium No. 16, ed. J. W. Evans (New York: Academic Press), 199.

Woolley, R. v. d. R., and Allen, C. W. 1948, M.N., 108, 292. 


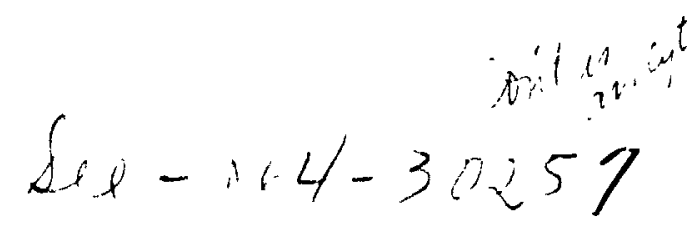

\title{
TIME VARIATIONS IN THE SOLAR EXTREME ULTRA- VIOLET SPECTRUM OBSERVED BY OSO-1*
}

\author{
W. M. NEUPERT
}

\section{INTRODUCTION}

A grazing incidence spectrometer' has beer flown as a pointed experiment on $\mathrm{OSO}^{-1}$ to monitor the extreme ultraviolct (EUV) spectrum of the sun. Over six thousand spectra were obtained at the rate of about 100 per day over a period of time from March through May 1962, corresponding to nearly three solar revolutions. Intermittent operation has subsecuently provided spectra for a period of greater than one year. This paper presents, in a condensed form, the variations observed in three of the more reliably identined lines of the spectrum: 284-A (Fe XV), 304-A (He II) and $335-\mathrm{A}$ (Fe XVI).

One may consider the solar EUV observations as falling into three categories: a) quiet corona, b) slowly varying components, and c) transient phenomena, such as flares. These categories are also listed in the order of increasing difficulty of making reliable observations. This paper will review some of the observations of slowly varying components that have been made and then present several instances in which transient phenomena have been observed.

\section{DESCRIPTION OF SPECTROMETER}

During operation the spectrometer was pointed at the center of the solar disk within approximately two min of arc. In this oientation, radiation from the entire solar disk and inner corona passed directly through the entrance slit and struck a cuncave grating mounted in grazing incidence, the angle of incidence being $88^{\circ}$. The exit slit and detector were mounted on a carriage

- Fublished a Goddard Space Flight Center Doeument X-61 4-64-24. Janung, 1004 which was driven on a circul " rail so that the exit slit scanned along the Rowland Circle, where the spectrum was focused, from $10-400-A$. The 50 micron entrance and exit a 'ts provided a spectral passband of 1.7-A and permitted resolution of lines $0.85-A$ apart. The detector was a windowless photomultiplier developed by the Bendix Corporation specifically for use in this spectrome: A tungsten photocathode was chosen to saluimize response to wavelengths above $1500-A$, and to reduce changes in sensitivaty due to variations of the emission properties of the cathude. The instrument calibration was performed (1) by exposing the entire spectrometer to a beam of monochromatic radiation of known intensity and (2) by evaluating the essential components of the spectrometer (grating, detector, etc.), and then computing the sensitivity of the instrument. The first method was used at 44-A using a proportional counter for determining the source intensity. The second method was applied at longer wavelengths $(80-400-A)$. In addition, a comparison was made of the solar fluxes obtained against the fluxes measured by Hall, Damon and Hinteregger ${ }^{2}$ with a calibrated rocket instrument. The comparison could only be made in the region of overlap, 250-A to $400-A$, and would be meaningful only if the solar radiation was the same. The 2800-Mc mean daily flux recorded by the National Research Council, Ottawa, Canada, vas used as an independent estimate of solar flux to choose the satellite data for the comparison. A hest fit was made between these methods of obtaining a calibration which yielded values of $8.0 \times 10^{8}$ photons $\mathrm{cm}^{-2}$ count $^{-1}$ at $335-\mathrm{A}$ and $4.2 \times 10^{5}$ photon $\mathrm{cm}^{-2}$ count $^{-1}$ at $284-A$. These values are useful for comparing the intensities of the lines at these two wavelengths, but their use to obtain absolute solar fluxes is not recommended. 


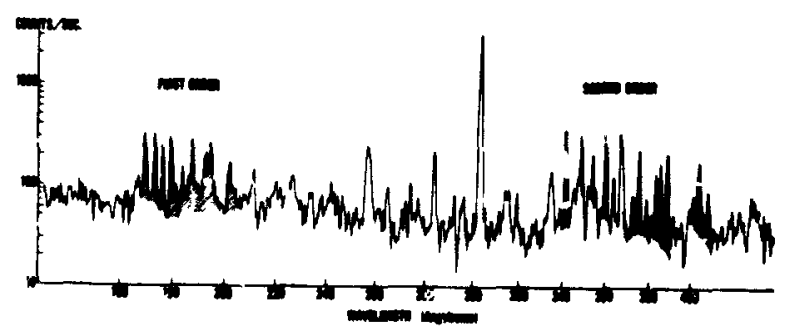

Figure 1.-Solar spactrum from $170 \mathrm{~A}$ to $400 \mathrm{~A}$ with second order line images indicated

\section{PRESENTATION OF DATA}

Figure 1 presents a typical scan obtained over the region of $170-A$ to $400-A$. Several factors reduce the usable spectral range of the spectroineter from its nominal range of $10-\mathrm{A}$ to $400-\mathrm{A}$. At wavelengths below 100-A the decreasing sensitivity of the spectrometer combined with an ircrease in scattered light upon approaching the central image made it impossible to distinguish a reliable spectrum on a single scan. However, a combination of several scans, using cross-correlation techniques, has shown the apparent existence of a line emission spectrum in the region from 50-A to 100-A (Neupert, Behring, White and Lindss $3^{2}$ ). At wavelengths above $342-\mathrm{A}$ the second order images of intense spectral lines observed in first order above 171-A obscure a considerable amount of interesting data. This effect is illustrated in Figure 1 which indicates, by crcsshatching, those first order lines which have strong second order counterparts in the recorded spectrum. These second order images were less prominent in earlier rocket results, leading to considerable dissimilarity between the rocket and satellite spectra above 342-A (Neupert and Behring ${ }^{4}$. For these reasons the obviously usable range of the instrument can be considered to be from 170-A to 340-A, a range which can be extended, with special precautiuns, to cover the interval from 50-A to $400-A$.

The brightest emission line in the region from 170-A to 340-A is the Lyman-alpha line of ionized helium at 304-A. In addition, numerous other emission lines appear with combir.ed flux comparable to, or sounewhat greater than, that of the helium line. Resonance lines of heavy ions $(\mathbf{M g}$ through $\mathrm{Fe}$ ) are expected in this region, leading to attempts (Zirin, Hall and Hinteregger'), (Neupert and Behring ${ }^{4}$ ) to identify the more prominent features of the spectrum in terms of such lines. The difficulty of positive identification can be appre eiated more if for example, we realize that the resonance lines of all stages of ionization of iron from $\mathrm{Fe} X$ through $\mathrm{Fe} X V I$, with the exception of $\mathrm{Fe} X V$, are expected in the small wa length interva! from $335-A$ to $390-A$. The overlapping of these resinance multiplets combined with second order radiation of shorter wavelengths makes positive identification a formidable task. The resonance lines of $\mathrm{Fe} X$ throngh Fe XIV have not been identified in the extreme ultraviolet (EUV) spectrum although their intensity as predicted by Ivanov-Kholodny and Nikolsky ${ }^{6}$ should permit their observation. Tousey ${ }^{7}$, working with a spectrum having higher resolution, reports that no $\mathrm{Fe}$ XIV multiplet can be found in his record. Because of these diñiculties, discussion will center around three of the more intense and more reliably identified lines of the spectrum, the Fe XV $\left(3 s^{2}{ }^{1} S_{0}-3 s 3 p{ }^{1} P 1_{1} \cdot{ }^{\circ}\right)$ line at 304-A. The location of these lines is indicated in 284-A, the Fe XVI $\left(3 \%^{2} S_{1 / 2}-3 p^{2} P^{2} \frac{3}{2}\right)$ line at 335-A, and the He II $\left(1 s-S_{1 / 2}-2 j^{2} P^{\circ} \frac{3}{2}, \frac{1}{2}\right)$ line at Figure 2.

\section{SLOWLY VARYING COMPONENTS OF THE EUV SPECTRUA?}

The months oi March and April of 1962, were auspicious for a study of the solur EUV spect:a in that oibservations could be made on both a quiescent and a disturbed solar atmosphere. During the second week in March the sun was especially quiet, the Zurich Provisional Relative runspot number being zero on 11 March. As the monih progressed solar rotation carried several enters of activity across the visible hemisphere of the sun. Definite enhancements in the solar spectrum were associated with these centers of activity.

Figure 2 presents two scans of the EUV spectrum taken approximately ten days upart. During the first of these observations (13 March, oniy one small region of activity was present on the solar cisk. In spite of this low level of nctivisy it is observed that the Fe XV an' $\mathrm{Fe} X V I$ lines persist as two of the mors prominent features of the spectrum. The recond spectrum (23 March) was obtained while several large and well-deveioped centers of activity were present on the risk. 


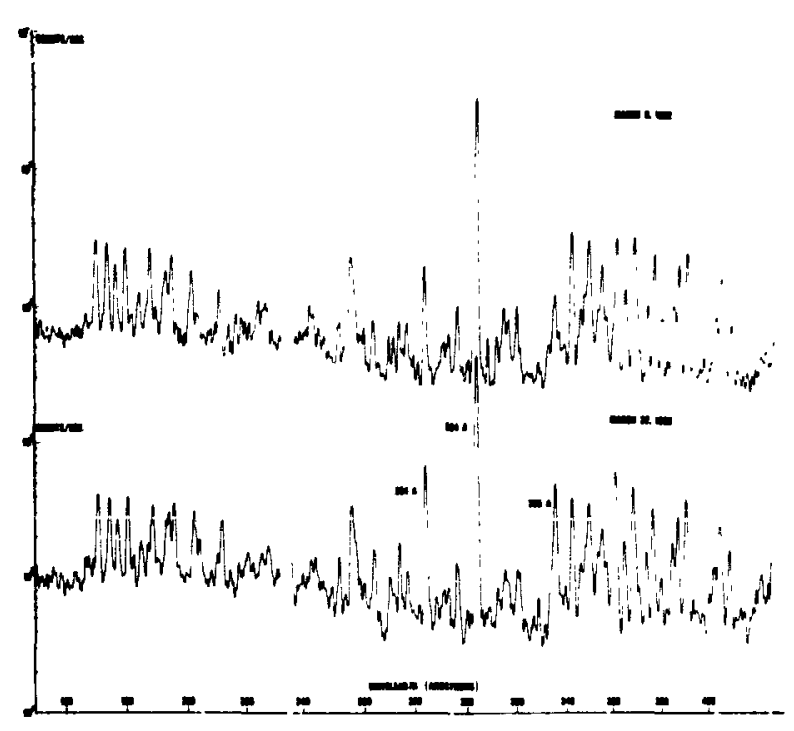

Figure 2.-Comparisou of iwo spuctral scans taken under different solar conditions

Comparing these two spectra we observe that the emission lines have increased in intensity but not all by the same amount. The Fe XV and XVI lines, s.lready prominent even in the near absence of solar activity, have increased in intensity appreciably more than any other line observed with certainty in this spectral range. The He II line has also in roased but by a lesser amount.

Time variations of these three lines as observed for the first 1066 orbits of operation are given in Figurs 3, in which each datum point represents

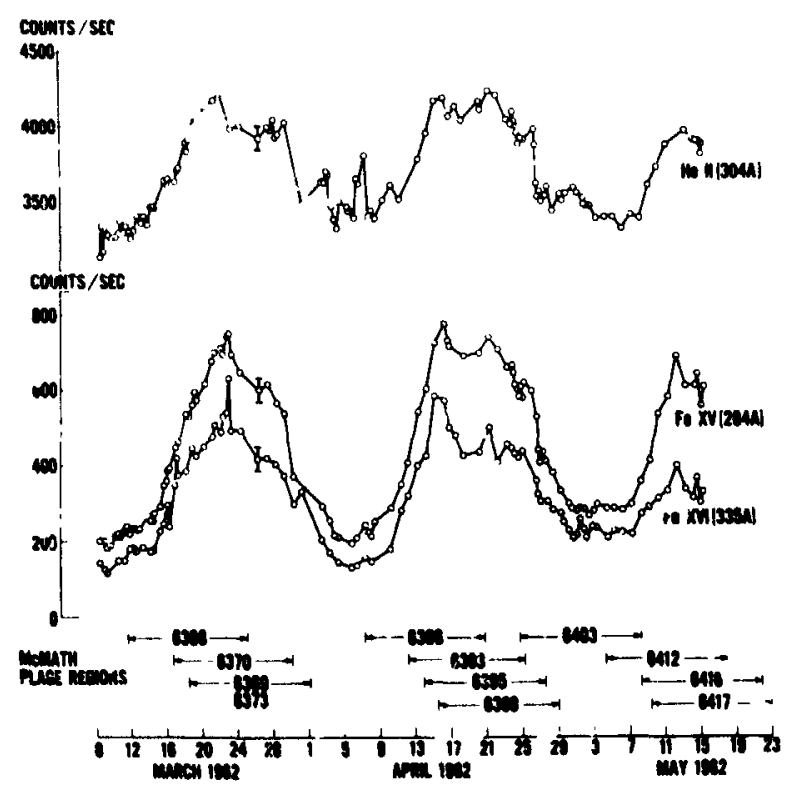

Fiaure 3. - OSO-1 data for three spectral lines compared with appearance and disappearance of major plage areas. the average of one orbit's observations (six to eight scans). Error bars indicating one standard deviation in the data are given for each wavelength. Also indicated are the times of appearance and disappearance of the major plage areas as cataloged by the McMath-Hulbert Observatory. The first increase in the counting rates above the "quiet sun" values is associated with the appearance of McMath Plage No. 6366 on the east limb on 11 March. The counting rate for the He II (304-A) line increases gradually to a maximum on 23 March, after which it drops rather abruptly to an apparent plateau. This sudden drop cannot be unambiguously attributed to the disappearance of plage region $\mathbf{6 3 6 6}$ on the west limb, for it follows quite directly after an unusual flare of importance 3 observed in plage region 6370 on 22 March, for which data are not. given in the figure. A gradual decline in readings is observed from 29 March to 7 April. A similar enhancement, followed by a return to near preplage rates, occurred in April. Since the spectrometer did not carry an internal standard it is impossible to state positively that its counting characteristies did not change over the period of observations. Tla consistent behavior of the data, hower $s$, as demonstrated in Figure 3, leads to the conclusion that no significant change in sensitivity occurred throughout the period of observation. (Subsequent analysis of real time data after one year in orbit supports this conclusion, even for the longor time interval).

Also shown in the figul 4 are the variations with solar activity of the coronal Fe XV (284-A) and Fe XVI (335-A) lines, the relative increases being considerably larger than for the He II (304-A) line. A definite, although sniall, enhancement associated with the appearance of plage No. 6366 is observed in both the Fe XVI and Fe XV lines. The possibility exists that the coronal emissions appear slightly earlier as might be expected since the coronal emission must take place at greater heights than the emission of ionized calcium radiation. The most obvious increase in intensity of these lines is associated with the appearance, in succession, of pluge areas numbered 6370,6369 and 6373 on 17 March through 19 March. The counting rates then increased gradually throughout this period indicating that the enhanced emission was not from a point source but rather from 
an extended volume having as its base an area at least as large as the underlying calcium plage. The disappearance of area 6366 on 25 March results in a slight decrease in counting rates, followed by a general decline from $29 \mathrm{March}$ through 3 April as the other plage areas are carried off the visible hemisphere of the sun by the solar rotation.

\section{DISCUSSION OF SLOWLY VARYING COMPONENTS}

The relationship of these observed counting rates to several ground-based measurements of solar activity is presented in Figures 4 and 6 . In Figure 4 the He II radiation is compared with

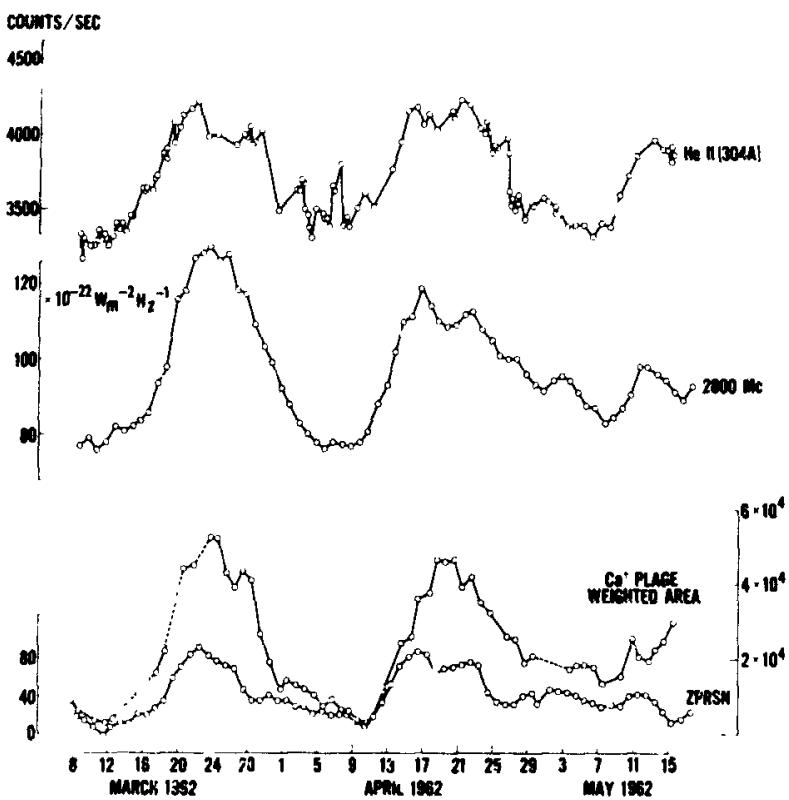

Figure 4.- Relationship of observed counting rates for the He II Lyman-alpha line to ground-based measurements of solar activity.

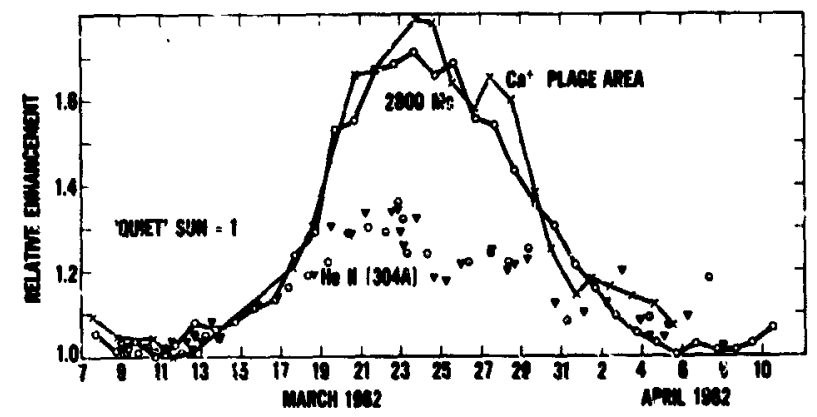

Flaure 5-Comparison of 304 A data, normalized to a "quiet sun" value of one, with weighted calcium plage data and $2800 \mathrm{Mc}$ radio observations.
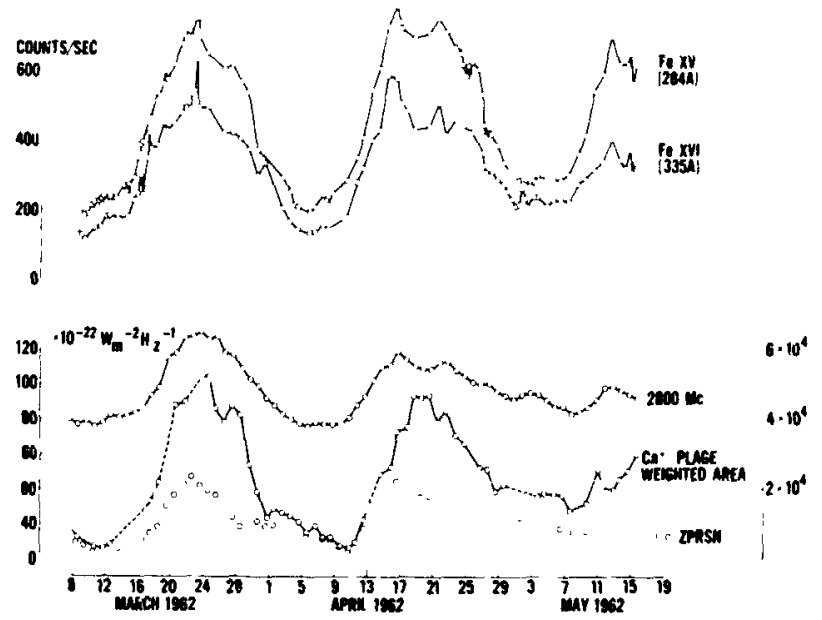

Figure 6.-Relationship of observed counting rates for the $284 \mathrm{~A}$ and $335 \mathrm{~A}$ line to ground-based observations.

daily values of the solar flux at $2800-\mathrm{Mc}$, and with the Zurich Provisional Relative Sunspot Number (ZPRSN). Also shown is an estimate of the calcium plage area, each area being weighted by the estimated intensity of the area on a scale from $i$ to 5 and by the cosine of its longitude from the central meridian. Values for this computation were supplied by the McMath-Hulbert Observatory.

A more detailed comparison is made in Figure 5 in which the radio and He II fluxes have been adjusted by dividing each observation by the rate observed for the "quiet sun." In addition, the weighted $\mathrm{Ca}^{+}$plage intensity has been adjusted to coincide with the $2800 \mathrm{Mc}$ enhancement on 21 March. This plot shows more clearly the close relationship which has been known for some time between the $2800 \mathrm{Mc}$ racliation and the plage areas and also shows the divergences of the He II (304-A) line from both of these. A better agreement between plages and the $304-\mathrm{A}$ line is obtained if only the plage areas, with no weighting factors for i.tensity or longitude from central meridian passage is used. In Figure 6 the ciuily values of siar Hux at $2800 \mathrm{Mc}$ and the Zurich Provisional Relative Sunspot Number are compared with the coronal lines of $\mathrm{Fe} X V(284-\mathrm{A})$ and $\mathrm{Fe}$ XVI (335-A). The estimatod weighted calcium plage area is also shown.

The spectral lines chosen for presentation here were selecied because they are reliably ideistified with perticular ions, not because they convey, more than any other line in the spectrum, changes 
in solar flux which occur with the appearance of plage areas. These three lines represent the extremes which have thus far been $\phi$ bserved in the spectral region from $171-\mathrm{A}$ to $400-\mathrm{A}$; only a few faint lines have smaller non-flare variations than the He II Lyman-alpha line, while no other outstanding lines have increases as great as those observed for 284-A and 335-A.

An attempt has been made to compute an electron temperature for the corona, as neariy as possible undisturbed by centers of activity, from the observed ratio of intensities of the Fe XV and Fe XVI lines. Such a calculation has validity only if both radiations originate in the same region of the corona at some well-defined electron temperature and only if an ionization-recombination equilibrium, as assumed by theory, is actually present. It is found that an electron temperature of $1.75 \pm 0.10$ million degrees in that part of the corona assumed to be the source of both ${ }^{r}$. XV and Fe XVI best fits the observed data. This result contrasts sharply with the value of 800,000 ${ }^{\circ} \mathrm{K}$ usually obtained from observation of the forbidden lines of $\mathrm{Fe} \mathrm{X}$ and $\mathrm{Fe} \mathrm{XIV}$.

It has been estimated that the emission of $\mathrm{Fe}$ $\mathrm{XV}$ and Fe XVI radiation essociated with a plage area is approximately 150 to 200 times that of an equivalent area of the quiet sun. The corresponding value for the 304-A line is 15 . Assuming that the increase in emission is due to a density increase only, one finds it necessary to hypothesice an increase in electron density by a factor of 12 to 14 over each plage for the two iron lines. The fact that a large range of intensit. variations is observed demonstrates, however, that an increase in electron density alone is not sufficient to explain the behavior of the spectrum. On the othe ${ }_{1}$ hand, the constancy of the ratio $\mathrm{Fe}$ $\mathrm{XVI} / \mathrm{Fe} \mathrm{XV}$ as one extrapolates to zero plage area argues against a significant change in electron temperature over plages. One can only conclude, at this time, that those regions (or region) of the corona emitting Fe XV and Fe XVI radiation occupy a relatively greater volume of the corona over plages and centers of activity.

The initial anulysis of only three lines (He II 304-A, Fe XV 284-A, Fe XVI 335-A) indicates that the relative prominence of spectral lines may depend upon the age of the center of activity which is responsible for the increased radiation.
As an example of this, one may observe (Figure 6) that the maximum emission in the $\mathrm{Fe} X V$ line apparently occurs later in time than the maximum for the $2800 \mathrm{Mc}$ flux, or for the plage areas, observed during March, April and May. In addition to such a slowly changing effect, one may note that localized perturbations appear (7-9 March and 16-17 April) for which the relative

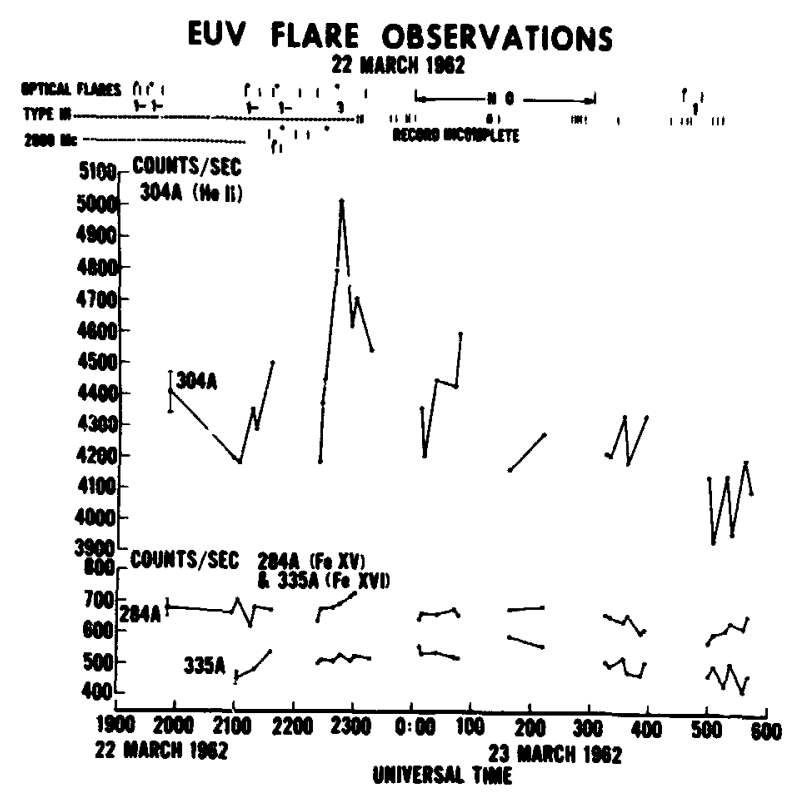

Figure 7a.-Increases in EUV line emission associated with optical flares.

EUV FLAKE OBSERVATIONS 13 MARCH 1962

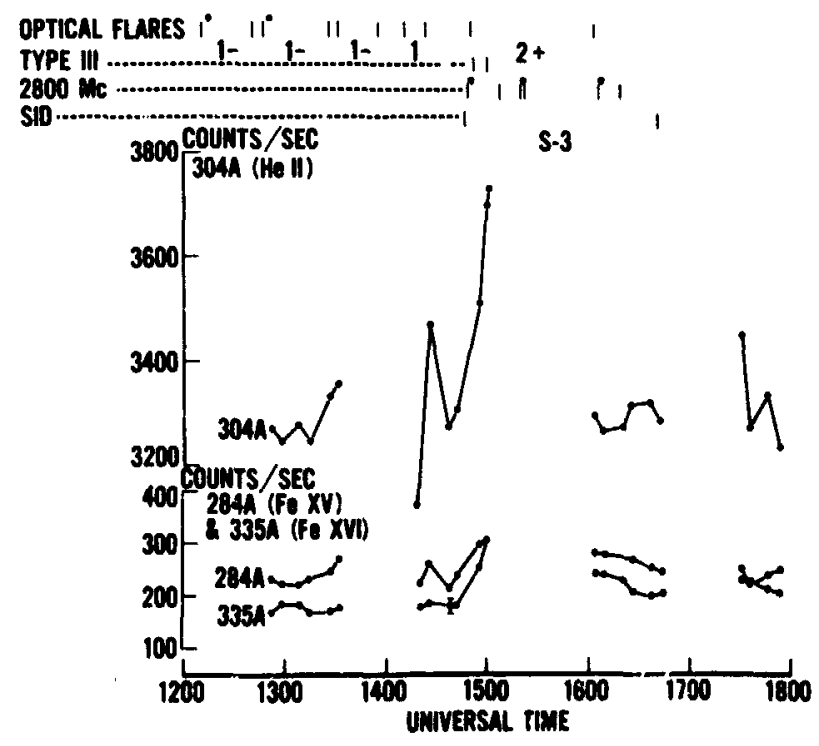

Frgure 7b.-Increases in EUV line emission associated with optical flares. 


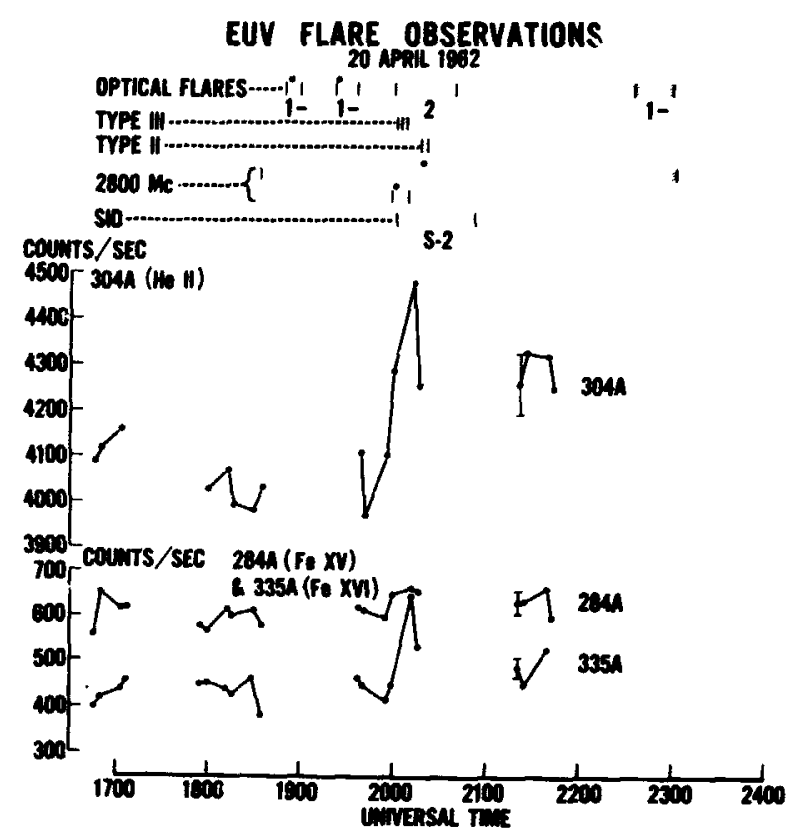

Figure 7c.-Increases in EUV line emission associated with optical flares.

increases in the helium and the iron lines are considerably different. In these instances we are apparently observing phenomena localized at particular levels in the solar atmosphere.

\section{EUV FLARE OBSERVATIONS}

It is dificult to obtain reliable EUV flare observations with the type of spectrometer used in OSO-1 because the flare radiation is seen as only a small increase in the radiation from the entire solar disk, and berause the long time required to scan the spectrum $(8 \mathrm{~min})$ severely limits the time resolution with which the flare can be observed. In spite of these difficulties, several increases in the EUV radiation have been associated with flares. Three such instances are shown in Figures 7 a,b,c. Supplem sntary information for these events is given in Table I.

\subsection{March, 1962 (Flare of Importance 3)}

From the observations of the $\mathrm{He}$ II line at 304-A made one minute before and three minutes after maximum in $\mathrm{H}$-alpha one may conclucle that the maximum at 304-A certainly occurs no earlier than $\mathrm{H}$-alpha maximum and may occur later. No changes at 284-A and 335-A can be associated - 'th this flare, optically of importance 3 but geophysically of much lesser importanc

TABLE 1.-Supplementary Flare Observations

A. 22 March 1962

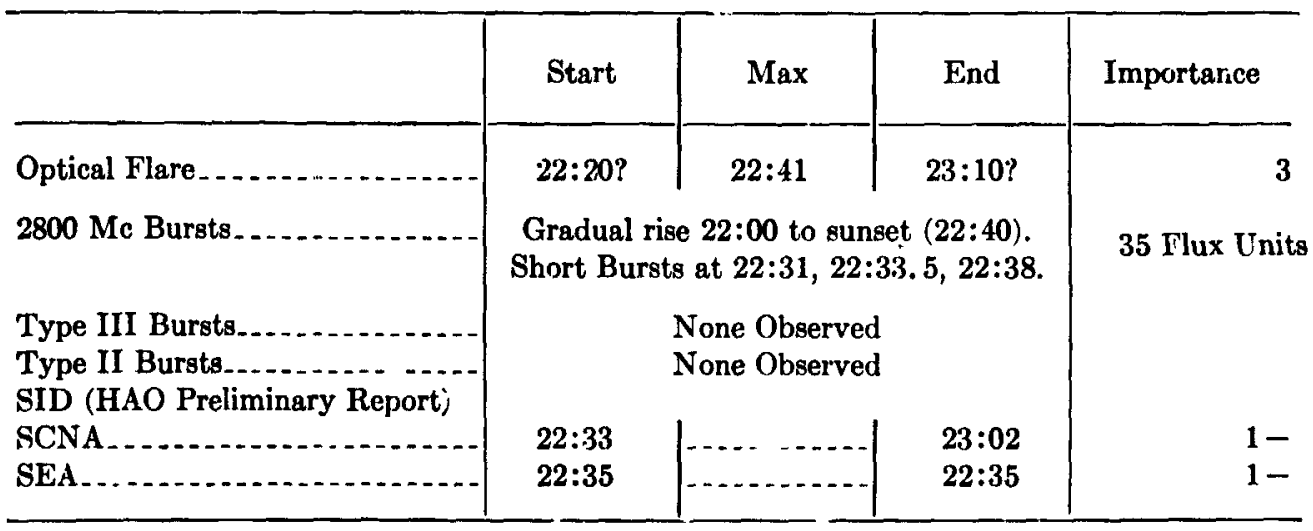

Approximate eUV Opgervation Times (Ut) At Peak of Flare March 22-23

\begin{tabular}{l|l|l|l|}
\hline & & (Peak) & \\
\hline $304 \mathrm{~A}(\mathrm{He} \mathrm{II}) \ldots \ldots \ldots$ & & \\
$284 \mathrm{~A}(\mathrm{~F}(\mathrm{XV}) \ldots \ldots$ & $22: 40: 23$ & $22: 44: 10$ & $22: 58: 00$ \\
$335 \mathrm{~A}(\mathrm{Fe} X \mathrm{XI}) \ldots \ldots$ & $22: 40: 03$ & $22: 43: 50$ & $22: 57: 40$ \\
& $22: 40: 53$ & $22: 43: 20$ & $22: 58: 30$ \\
\hline
\end{tabular}


TABLE 1.-Supplementary Flare Observations-Continued

B. 13 March 1962

\begin{tabular}{|c|c|c|c|c|}
\hline & Start & $\operatorname{Max}$ & End & Importance \\
\hline Optical Flare & $\begin{array}{c}14: 48 \\
\text { (earlier than) }\end{array}$ & $15: 05$ & $\begin{array}{c}16: 01 \\
\text { (later than) }\end{array}$ & $2+$ \\
\hline SID (CRPL-F Bulletin, NBS) & & & & \\
\hline $\begin{array}{l}\text { SCNA } \\
\text { SEA }\end{array}$ & $\begin{array}{l}14: 50 \\
14: 50\end{array}$ & $15: 00$ & $\begin{array}{l}15: 45 \\
15: 55\end{array}$ & $\begin{array}{l}1 \\
2\end{array}$ \\
\hline SPA & $14: 45$ & $15: 05$ & $16: 40$ & \\
\hline 2800 Mc Bursts & $14: 47.5$ & $14: 50.5$ & $15: 07$ & 470 Flux units \\
\hline Type III Bursts & $14: 50$ & 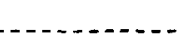 & $14: 58$ & Group of Bursts \\
\hline
\end{tabular}

Approximate EUV Observation Times (UT)

\begin{tabular}{l|r|r|r|r}
\hline & & & & (Peak) \\
\hline $304 \mathrm{~A}(\mathrm{He}$ II) & & & \\
$284 \mathrm{~A}(\mathrm{Fe} X V)$ & & & \\
$335 \mathrm{~A}(\mathrm{Fe} X V I)$ & $14: 40: 05$ & $14: 53: 55$ & $14: 57: 10$ & \\
\end{tabular}

C. 20 April 1962

\begin{tabular}{|c|c|c|c|c|}
\hline $\begin{array}{l}\text { Optical Flare } \\
\text { SID (CRPL-F Bulletin NBS) }\end{array}$ & $19: 58$ & $20: 02$ & $20: 40$ & $2+$ \\
\hline STO (CRPL-F Bulletin, NBS) & & & & \\
\hline $\begin{array}{l}\text { SCNA } \\
\text { SEA }\end{array}$ & 20:00 & $20: 04$ & $20: 35$ & 2 \\
\hline $\begin{array}{l}\text { SEA } \\
\text { SPA }\end{array}$ & $20: 01$ & - nen & $20: 50$ & 2 \\
\hline SPA__. & $20: 02$ & 20:07 & $20: 50$ & $\ldots 2$ \\
\hline 2800 Mi Burst_....... & $19: 57.3$ & $19: 59$ & $20: 09$ & 80 Flux Units \\
\hline Type III Bursts & 20:00. 6 & $\cdots$ & 20:02 & (Harvard) \\
\hline & 20:00 & & $20: 04.15$ & (HAO) \\
\hline & $20: 05.15$ & $\ldots$ & $20: 07.30$ & (HAO) \\
\hline Type II Bursts......... & $20: 04.6$ & $-\ldots$ & $20: 19$ & (Harvard) \\
\hline & $20: 16$ & & $20: 20.3$ & $(\mathrm{HAO})$ \\
\hline
\end{tabular}

Approximate EUV Observation Times (UT)

\begin{tabular}{|c|c|c|c|}
\hline & & (Peak) & \\
\hline $\begin{array}{l}\text { 304A (He II) } \\
\text { 284A (Fe XV) } \\
335 \text { A (Fe XVI) }\end{array}$ & $\begin{array}{l}19: 58: 00 \\
19: 58: 20 \\
19: 57: 30\end{array}$ & $\begin{array}{l}20: 11: 50 \\
20: 11: 30 \\
20: 12: 20\end{array}$ & $\begin{array}{l}20: 15: 00 \\
20: 15: 20 \\
20: 14: 30\end{array}$ \\
\hline
\end{tabular}




\subsection{March, 1962 (Flare of Importance 2+)}

Peak phase of this flare apparently occurred near satellite sunset. The last valid observations before sunset indicatc that the LUV flare radiation reaches a maximum after the $2800 \mathrm{Mc}$ radio burst. Increases are noted for all three lines, with the ratio $335-\mathrm{A} / 284-\mathrm{A}$ apparently increasing at or near maximum phase of the optical flare. The enharcement in the coronal lines may continue after cessation of the visible flare.

\subsection{April, 1962 (Flare of Importance 2+)}

EUV observations are poorly situated in time relative to the optical maximum of this flare. It appears, therefore, that the EUV emission maxima may have been significantly greater than the maximum reading actually recorded. In spite of this shortcoming, an increase in the Fe XVI radiation, relative to Fe XV can again be observed.

\section{DISCUSSION OF FLARE OBSERVATIONS}

A considerable number of additional events have apparently been observed, some associated with optical flares; others not. However, the data for these events have not been sufficiently analyzed to establish their validity. The increase of the ratio $\mathrm{Fe} \mathrm{XVI/Fe} \mathrm{XV} \mathrm{is} \mathrm{inost} \mathrm{frequently}$ associated with sular events producing ionospheric disturbances. The increase in this ratio suggests that some volume of the solar atmosphere, associated with the flare, has been raised to a higher electron temperature.

It is interesting to note that the EUV maxima coincide more closely with the optical maxima and with ionospheric affects than they do with centimetric radio bursts. The association with ionispheric effects is understandable if we assume that the hard X-rays, which themselves may be line emission at short wavelengths, and which are causing these ionispheric disturbances, have the same common thermal origin in flares as does EUV flare radiation observed by the OSO-1 spectrometer. On the other hand, the coincidence with optical $\mathrm{H}$-alpha maxima cannct be explained in the same manner since the hydrogen radiation must originate in a cooler region of the solar atmosphere.

\section{DISCUSSION}

Maron: In comparing the active sun and the quiet sun, is your finding that the variations in fluxes from the lines in the higher ionization states of iron hava greater amplitude than the lower states? Is this not most simply interpreted as saying that the relative density change is greatest in the regions of highost temperature?

Dr. Neupert: Yes, that is a possible conclusion. However, we cannot discriminate between this hypothesis and one in which the clectron temperature profile of the corona changes above the active region in such a manner that a greater relative volume is at the higher temperature.

Zirim: Just oxe remark. I have the impression that you have essentially a factor of two increase in the total radiation from the whole sun coming from a flare which essentially is .1 percent of the area, or something like that, which impresses me with a very large change. Is that so, would you say?

Dr. Neupert: We can associate changes of 50 percent or more during flares only with the $\mathrm{Fe}$ $\mathrm{XVI}$ and Fe XV lines. Many lines show no statistically reliable change. For the event on March 13 we can demonstrate, by averaging our data over one complete orbit, that several other lines do show changes. Rapid variations of 10 percent to 20 percent in the faint lines would be unobservable because of statistical fluctuations. We should probably use the $\mathrm{H}$-alpha area of a flare only as a lower limit in estimating the size of the EUV emitting region.

Guerjoy: Would you clarify for me how long, how large an area of the sun you are lonking at?

Dr. Neupert: We are looking at the eittire solar disc and out to about a quarter of a solar radius from the limb. So we are looking at the entire disc plus the inner corona with this instrument.

Chubb: Can you say something about how the variability of the lines around 170 angstroms is as compared to. say, the 304 and the iron lines?

Dr. Neupert: The variations with solar plage area of the lines between $170-A$ and $200-A$ are less than those of the 284-A and 335-A lines. They are comparable to or somewhat more than that of the helium line. We have not analyzed flare data for these lines but it is our experience that lines which increase most due to the presence 
of a center of activity also increase the most during flares.

Dr. Lindsay: I note that you have averaged about 16 lines in this region over a period of about a month, as I recall. Why don't you report at least the integrated or the average of these lines varies?

Dr. Neupert: We have made integrations over the spectrum for two days. Not for the 13th of March and 23rd of March, but for two days on which activity was similar to those which we had in the second slide. The increase in counting rate was of the order of 50 to 80 percent. This is an increase due to the presence of centers of activities on the sun's disc, not increase due to flares.

\section{REFERENCES}

1. W. E. Benring, W. M. Neuperi and W. A. Nichols, J. Opt. Soc. Amer. 52 (5) :597, May, 1962.

2. L. A. Hall, K. R. Damon and H. E. Hinteregger, Space Research III, ed. W. Priester (Amsterdam, North-Holland Publ. Co., 1963) 745.

3. W. M. Neupert, W. E. Behring, W. A. White and J. C. Lindsay, GSFC Document X-614-62-156 (1962)

4. W. M. Neupert and W. E. Behring, J. Quant. Rad. Transf. 2 (1962) 527.

5. H. Zirin, L. A. Hall and H. E. Hinveregger, Space Research III, ed. W. Priester (Amsterdam, NorthHolland Publ. Co., 1963) 760.

6. G. S. Ivanov-Kholodny and G. M. Nikolsky, Astronomicheskii Zhurnal 38 (1961) 828.

7. R. Tousey, Space Science Reviews (to be published) (1963). 


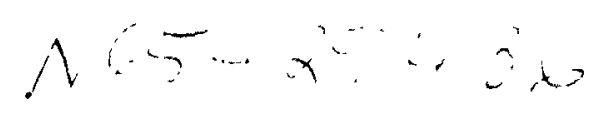

\title{
THE UV LINES OF FE XIV IN THE SOLAR CORONA
}

\author{
, R. STOCKHAISEN
}

Knowledge of the structure of the solar corona can be increased by observations of the many emission lines in the far ultraviolet. Hower er, identification of these lines is made difficult by the lack of knowledge of many of the atomic parameters relevant to these lines. Garstang's (1962) calculations of energy levels and oscillator strengths for Fe XIV allow predictions of lines strengths and wave lengths for the lines of this ion.

Collision cross-sections of the optically permitted lines are related to the oscillator strengths by the expression given by Van Regeınorter (1962)

$$
Q(n, m)=\frac{1}{k_{n}^{2}} \frac{8 \pi}{\sqrt{3}} \frac{I_{H}}{\Delta E} f(n m) g \pi a_{o}^{2}
$$

For levels not connected to the ground level by an optically allowed transition, electron exchange collisions are the only excitation mechanism. in a manner similar to Osterbrock (1963) these exchange cross-sections can be estimated by

$$
\begin{aligned}
Q_{e}(i n, j m)<Q_{0}(i n, j m) \\
=\frac{\pi}{k_{n}^{2}}\left\{\begin{array}{l}
(2 \ell+1)\left[g_{j, m} / \underset{m}{\Sigma} g_{j m}\right] \\
\left(2 \ell^{\prime}+1\right)\left[g_{j m} / \Sigma_{m} g_{j m}\right]\left(g_{j} / g_{t}\right)
\end{array}\right. \\
\text { or } \frac{\pi}{\mathrm{k}_{n}^{2}}\left\{\begin{array}{l}
(2 \ell+1) \\
\left(2 \ell^{\prime}+1\right)\left(g_{j m} / g_{i n}\right)
\end{array}\right.
\end{aligned}
$$

whichever of the four is smaller. Here $i n$ is the $n^{i n}$ level of term $i$ and $j m$ is the $m^{i n}$ level of term $j$. The term in square brackets comc 3 from the assumption that the levels of term $j$ are populated in proportion to this weight factor.

In his work with nelular ions, Osterbrork. has taken $Q_{0}=0.10_{0}$. On the basis of the rapid decrease, wish increasing energy of the colliding

*Publinhad as Goddard Space Flioht Center Doetimont X-310-64-808. July 1964. electron, of the cross-sections for exchange collisions in He I (St. John, Miller, and Lin 1964) and the decrease of exchange cross-sections along the iso-electronic sequences shown in Table VIII of Seaton (1958), Dr. Osterbrock has suggested that a value of $Q_{c}$ nearer to $Q_{e}=0.01 Q_{0}$ may be more appropriate for $\mathrm{Fe}$ XIV. With the uncertainties involved, parallel calculations were made under three assumptions:

$$
Q_{e}=\left\{\begin{array}{l}
.0 \\
.1 Q_{0} \\
.01 Q_{0}
\end{array}\right.
$$

The total cross-section is then the sum of (1) and (3).

Having the collision cross-sections, production rates of the UV lines can be calculated. Following the notation of Seaton (1963), the rate of excitation from level $i$ to level $j$ followed by spontaneous transition to level $k$ is given by

$$
\alpha_{i k}=q_{i} \Gamma_{j k}
$$

where $q_{i j}$ is the collisional excitation rate of $j$ from $i$ and $C_{j k}$ is the probability of going to $k$ from $j$. For the UV lines of Fe XIV, $C_{j k}$ does not involve coliisional de-excitation due to the high spontaneous transition probabilities, and the low coronal electron densities.

Table 1 gives the production rates for UV lines due to transitions ending ir the two levels ( 1 denotes ${ }^{2} P^{\circ}{ }_{1 / 2}$ and 2 denotes $\left.{ }^{2} P^{\circ}{ }_{3 / 2}\right)$ of the ground term. Results are given for two temperatures and three values of the exchange cross-section. Excitations are assumed to take place only from the ground level.

The sum of the production rates to the upper level $\left({ }^{2} P^{\circ} \mathrm{a} / 2\right)$ of the ground term is impoitant in that it gives the excitation rnte (due to cascade) of the coronal green line, $\lambda 5303$. It can be seen that there is a slight discrepancy between these 
TABLE 1.-Production Ratcs

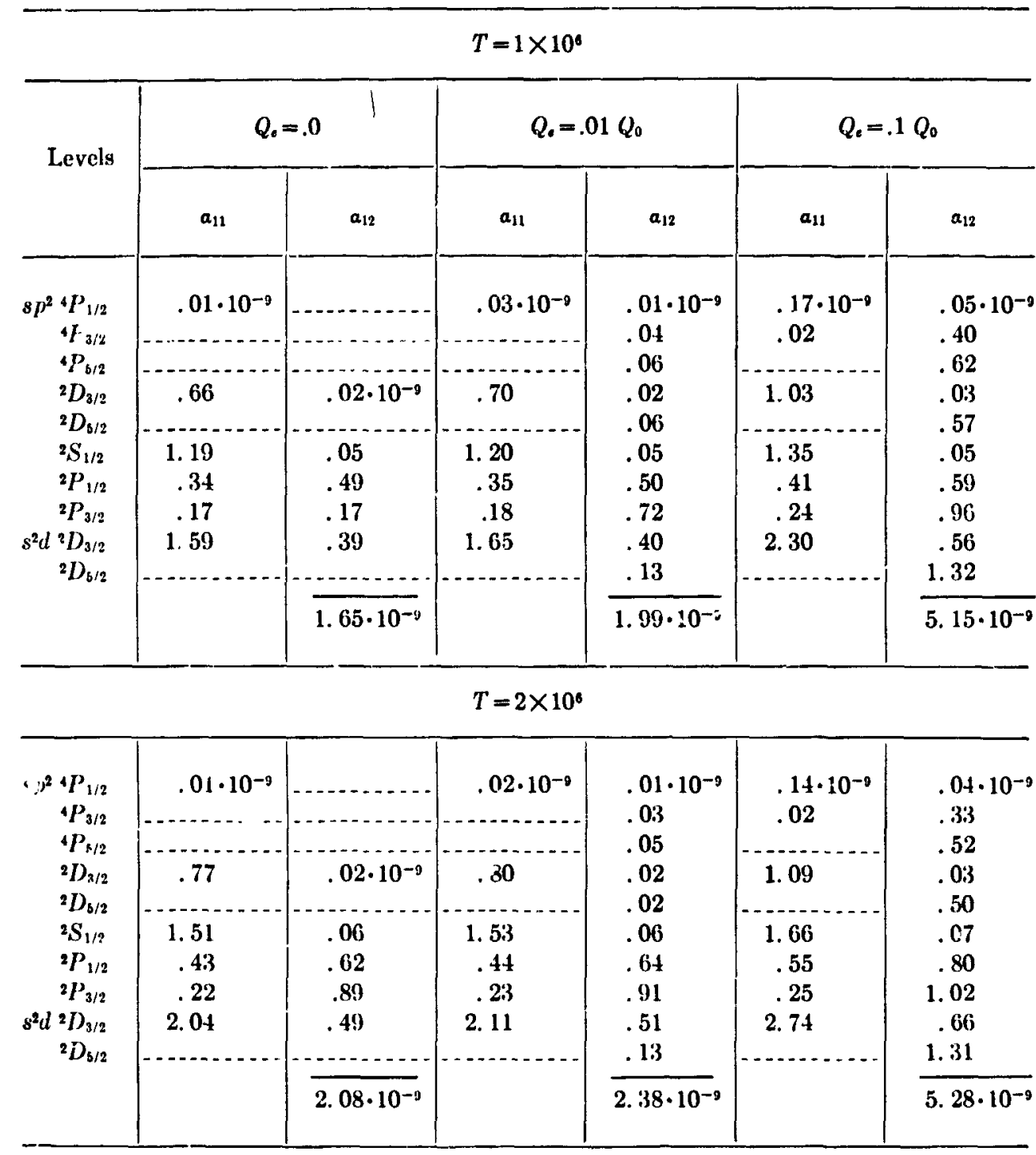

cascade excitation rates and those given in Table 6 of Seaton (1963). In a private communication, Dr. Seaton has noted a numerical eror in his work.

Inclusion of the exchange collisions has a significant effect on the production rates only for those levels which have low $f$ values with respect to the ground level, and then on!y for the case $Q_{0}=0.1 Q_{0}$.

The product of these production rates and the energy of the respective emitted photons, normalized so that the ${ }^{2} P^{\mathrm{C}_{1 / 2}}-{ }^{2} P_{3 / 2}$ transition is unity, gives the predicted relative line strengths of the expected UV lines. The se strengths are given in Table 2. Table 3 gives the predicted wavelengths and the wavelengths aseigned hy
Neupert and Smith (1964) from observations of the NASA satellite OSO-I. These identifications were made on the basis of time variations of the line strengths and wavelength separations of the i unltiplet members. Also included are the observed relative line strengths for two periods of observation in 1962. Quict Sun (average of March $8,9,10$ ) is a period of low solar activity and April 20 is a period $n f$ high solar activity. These observed line strengths are also normalized to the ${ }^{2} P_{1 / 2}^{\circ}-{ }^{2} P_{3 / 2}$ line. This line was about 1.5 times more intense on April 20 than during the Quiet Sun period. At this stage of the $r$ 'uciions, the observed relative line strengths are accurate to only a few tenths (in these units). 
TABLe 2.-Predicted Linc Strengths

\begin{tabular}{|c|c|c|c|c|c|c|c|c|}
\hline \multirow[b]{3}{*}{$3 s^{2} p-3 s p^{2}$} & \multirow{3}{*}{$J_{L}$} & \multirow{3}{*}{$J_{U}$} & \multicolumn{6}{|c|}{$I$} \\
\hline & & & \multicolumn{3}{|c|}{$T=1 \times 10^{6}$} & \multicolumn{3}{|c|}{$T=2 \times 10^{8}$} \\
\hline & & & $Q_{1}=.0$ & $Q_{.}=.01 Q_{0}$ & $Q_{1}=.1 Q_{0}$ & $Q .=.0$ & $Q_{0}=.01 Q_{0}$ & $Q_{e}=.1 Q_{0}$ \\
\hline \multirow[t]{5}{*}{$P^{0}-4 P_{\ldots} \ldots$} & $1 / 2$ & $1 / 2$ & $\ldots$ & .1 & .5 & & & .3 \\
\hline & $1 / 2$ & $3 / 2$ & & $\ldots$ & & & & \\
\hline & $3 / 2$ & $1 / 2$ & & $\ldots$ & .1 & & & .1 \\
\hline & $3 / 2$ & $3 / 2$ & & .1 & .8 & & .1 & .7 \\
\hline & $3 / 2$ & $5 / 2$ & $\ldots \ldots \ldots$ & .2 & 1.4 & & .1 & 1. 1 \\
\hline \multirow[t]{3}{*}{${ }^{2} P^{0}-2 D \ldots \ldots$} & $1 / 2$ & $3 / 2$ & 2.8 & 2.8 & 3.1 & 2.5 & 2.5 & 3. 2 \\
\hline & $3 / 2$ & $3 / 2$ & .1 & .1 & .1 & .1 & .1 & .1 \\
\hline & $3 / 2$ & $5 / 2$ & $\ldots \ldots \ldots$ & .2 & 1.6 & $\ldots$ & .1 & 1.4 \\
\hline \multirow[t]{2}{*}{$2 P^{0}-2 S \ldots \ldots$} & $1 / 2$ & $1 / 2$ & 6.4 & 6.1 & 5.2 & 6. 3 & 6. 1 & 6. 1 \\
\hline & $3 / 2$ & $1 / 2$ & .3 & .2 & .2 & .2 & .2 & .2 \\
\hline \multirow[t]{4}{*}{$2 P^{\circ}-2 P$} & 1,2 & $1 / 2$ & $2:$ & 1.9 & 1.7 & 1.9 & 1.9 & 2. 2 \\
\hline & $1 / 2$ & $3 / 2$ & .0 & 1.0 & 1.0 & 1.0 & 1.0 & 1.0 \\
\hline & $3 / 2$ & $1 ; 2$ & 2.7 & 2.6 & 2.3 & 2.6 & 2.6 & 3.0 \\
\hline & $3 / 2$ & $3 / 2$ & 3.9 & 3.8 & 3.8 & 3.9 & 3.8 & 3.9 \\
\hline \multirow{4}{*}{$\begin{array}{l}3 s^{2} p-3 s^{2} d \ldots \ldots \\
2 P^{\circ}-1 D \ldots \ldots\end{array}$} & & & & $\ldots$. & $\ldots$. & 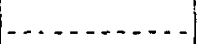 & $\ldots$. & $\ldots$ \\
\hline & $1 / 2$ & 3,2 & 10.9 & $10 . \%$ & 11.2 & 10.8 & 10. 7 & 12.8 \\
\hline & $3 / 2$ & $3 / 2$ & 2.6 & 2.5 & 2.6 & 2.5 & 2.5 & 3.0 \\
\hline & $3 / 2$ & $5 / 2$ & $\ldots \ldots$ & .8 & 6. 2 & $\ldots \ldots$ & .6 & 5. 9 \\
\hline
\end{tabular}

TaBde 3.-Observed Relative Intensitics and Watelengths

\begin{tabular}{|c|c|c|c|c|c|c|}
\hline $\begin{array}{r}\text { Ievels } \\
3 s^{2} p-3 s p^{2}\end{array}$ & $J_{L}$ & $J_{v}$ & $\lambda_{P}$ & $\lambda_{v}$ & $\begin{array}{c}l \\
\text { Quiet sun }\end{array}$ & April 20 \\
\hline & $1 / 2$ & $1 / 2$ & 272.9 & 274.2 & 1.1 & 1.9 \\
\hline & $3 / 2$ & $1 / 2$ & 287.7 & 288.6 & .6 & .6 \\
\hline \multirow[t]{4}{*}{$2 P^{0}$} & $1 / 2$ & $1 / 2$ & 256.0 & Blend & $\ldots$ & $\ldots$ \\
\hline & $1 / 2$ & $3 / ?$ & 250.5 & 251.8 & 1.0 & 1.0 \\
\hline & $3 / 2$ & $1 / 2$ & 269.0 & 270.5 & .7 & 1.0 \\
\hline & $3 / 2$ & $3 / 2$ & 262.9 & 264.5 & 1. 2 & 1.5 \\
\hline \multirow{4}{*}{$\begin{array}{l}38^{2} p- \\
2 p^{0}\end{array}$} & & & & & & 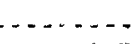 \\
\hline & $1 / 2$ & $3 / 2$ & 214.1 & 203.9 & .9 & 1.3 \\
\hline & $3 / 2$ & $3 / 2$ & 223. 1 & $2 j 15$ & 1.3 & 18 \\
\hline & $3 / 2$ & $5 / 2$ & 222.0 & & & \\
\hline
\end{tabular}

In comparing the observations and predictions, it must be noted that the satellite observations do not cover the spectral region occupied by the ${ }^{2} P^{\circ}-4 P$ multiplet. For the $s^{2} P\left({ }^{2} P^{\circ}\right)$ multiplet, $-s p^{2}\left({ }^{2} D\right)$ no lines of the predisted strengths or with the co time behavior appear to be present at tut predicied wavelengihs, however the instrumental sensitivity is decreasing in this region.

Since these obscrved strengths are a result of contributions from the entire solar dise, and due to the uncertainties in the daia, only semi-quantitative comparisons with the calculated strengths will be made. Most apparent is the rciatively large spreal in predicted strengths as compared 
to the nbserved strengths. Using a temrerature of $5 \times 10^{5}$ does not significantly reduce this spread. Exchange collisions do not seem important for most cases. However, for the $s^{2} p\left({ }^{2} P^{\circ}\right)-s^{2} d\left({ }^{2} D\right)$ multiplet the ratio of the ratio of the sum of the predicted intensities of the $3 / 2-3 / 2$ and $3 / 2-5 / 2$ lines to the $1 / 2-3 / 2$ line (ratio $=8.8: 11.2$ for $T$ $=10^{6}$ and $Q_{0}=0.1 Q_{0}$ ) is closer (but still in the wrong sense) than for the case where $Q_{0}=0$.

The possibility of blends cannot be ruled out absolutcly, but since the time variations of all these line strengths was quite similar, it is assumed that blends have a small effect. One possibility for the discrepancies in relative strengths is some kind of self-absorption. Due to the very low rate of collisional de-excitation (relative to spontaneous transitions), "true" absorption is not to be expected. Repeated reabsorptions of the UV photons in the corona can shange the relative strengths since after each absorption the atom has a choice of making a transition to either of the ground levels. This has the effect of making the relative strengths more nearly equal, which is the desired effect in this case.

This effect has been discussed for the corona by Pecker and Thomas (1963) and Pottasch (1964). Since the number of times a photon is scattered in leaving the corona is $\tau^{2}$, the optical depth must be greater than unity to have any effect at all. Pottasch (1964) has calculated cptical depths for some of the transitions involved here using an electron density of $\left.2 \times 10^{8} \mathrm{~cm}^{-3}\right)$. These values $\left(r \approx 10^{-1}\right.$ to $\left.10^{-2}\right)$ are too small to have any effect on the relative line strengths.

Neupert, Behring and Lindsay (1963) have shown that the UV line strengths seem to be correlated with plage regions and other solar activity. It may be that a higher electron density than that used in Pottasch's calculations may be more appropriate. However, even the electron densities measured by Aly, Evans, and Orrall (1962) in a bright coronal condensation $\left(\leq 3 \times 10^{\circ} \mathrm{cm}^{-3}\right)$ are too small to give self-absorption for most of the lines. One possible solution to the problem would be an inhomogeneous corona in which the dense elements had electron densities in t'ie neighborhood of $10^{10} \mathrm{~cm}^{-3}$.

There is one other interesting observation from OSO-1 that needs to be explained. The UV line intensities vary by a factor of two or three for different conditions of solar activity, while the intensity ratios vary by about $\mathbf{5 0}$ percen ${ }^{+}$or less. In terms of an inhomogeneous model, th. 3 might be explained as each dense element being optically thick (thereby giving the correct intensity ratios). An increase in solar activity would merely mean an increase in the number of dense elements (thereby increasing the intensities of the lines, hat, having less effect on the intensity ratios. Further reduction of data and calculations should allow a more quantitative discussion of the observations

I am grateful to W. Neupert and E. Smith for discussions of the observations and for the relative line strengths prior to publication, to S. Kastner for br!pful discussions and to D. Osterbrock ${ }^{\circ}$ or several discussions, especially those concer. ag the exchange cross-sections and the self-absorption mechanism.

\section{REFERENCES}

Aly, M. K., Evans, J. W., and Orkale, F. W. 1962, Ap. J., 136, 956.

Garstanci, R. H. 1962, Ann. d'ap., 25, 109.

Neupert, W. M., Behring, W. E., and Lindsay, J. C. 196i3, Faper given at the Fourth International Space Science Sympreium, Warsaw, Poland.

Neupert, W. M., and Smith, E. v. P. 1964, Paper given at June 24-27 meeting of the Amerisan Astronomical Society.

Osteraruck: D. E. 1063, Plinet, Space Sci., 11, 621.

Pecker, C., and Thomas, R. N. 1963, Ap. J., 137, 967.

Porta:ten, S. R. 1964, M. N. R. A. S., 128, 73.

Recigmohten, H. van. 1962, Ap. .., 136, 106.

SE...ov, M. J. 1958, Kev. Mod. Phys., 30, 979. 1963, Planel, Space Sci., 12, 55.

St. Jor.n, R. M., MrLler, I. L., and Lis, C. C. 1064, Phys. Rev., 134, A888. 